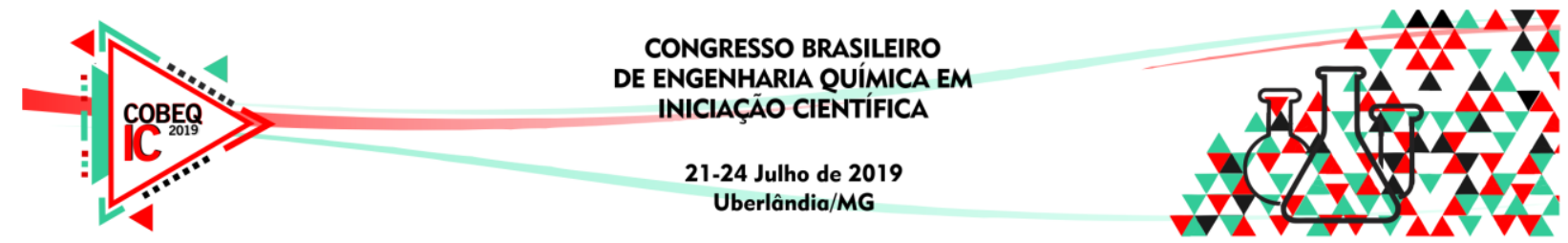

\title{
MODELAGEM MATEMÁTICA DO PROCESSO DE SECAGEM DE RESÍDUOS AGROINDUSTRIAIS
}

\author{
L. D. SILVA ${ }^{1}$, G.M. ANDRADE ${ }^{1}$, H. A. C. NERE ${ }^{1}$, O.S. PEREIRA ${ }^{2}$ e C. S. S. PEREIRA ${ }^{2}$ \\ ${ }^{1}$ Universidade de Vassouras, Engenharia Química \\ ${ }^{2}$ Universidade Federal Rural do Rio de Janeiro, Departamento de Matemática \\ E-mail para contato: larilarissadiasxd@gmail.com
}

\begin{abstract}
RESUMO - Os resíduos gerados no processamento de frutas pelas indústrias alimentícias são ricos em nutrientes que podem ser reaproveitados na alimentação humana ou na fabricação de ração animal, constituindo-se em uma alternativa para evitar o desperdício. O processo de secagem é utilizado como método de conservação para redução da deterioração e perdas de produtos. Devido à importância industrial, comercial e ambiental do reaproveitamento de resíduos agroindustriais, o presente trabalho avaliou a cinética de secagem dos resíduos de tomate, laranja e mamão, gerados por uma indústria da região. Para o ajuste das curvas cinéticas experimentais utilizou-se o modelo matemático de Page. Os cálculos e a construção das curvas de secagem foram realizados com o auxílio do Excel e as constantes do modelo foram estimadas através da ferramenta Solver. A modelagem utilizada descreveu de forma satisfatória os dados de secagem dos resíduos avaliados.
\end{abstract}

\section{INTRODUÇÃO}

O consumo das frutas tropicais é geralmente in natura, visto que sua apreciação nestas condições evidencia melhores características de cor, textura, aroma e propriedades nutricionais. Não obstante, tratando-se de produtos perecíveis, em sua grande maioria são processados e tornam-se produtos como sucos, néctares, polpas, geleias e doces (Infante et al., 2013).

Souza (2011) explicita que o Brasil é um dos países que mais produz resíduos agroindustriais, as indústrias de polpas são um exemplo da geração de resíduos de frutas, colaborando no crescimento da produção do lixo orgânico. Com a ampliação da produção industrial, a degradação do meio ambiente torna-se um assunto prioritário. Esses resíduos poderiam ser aproveitados, minimizando o desperdício de alimentos e gerando uma nova fonte alimentar.

Segundo Infante (2013), as frutas após o processamento geram subprodutos, não possuindo um destino inerente tornam-se contaminantes ambientais, como resultado o tratamento para descarte gera custos operacionais às empresas. Sabendo que esses resíduos agroindustriais são capazes de atuar como antioxidantes naturais, sua aplicação poderia ser introduzida na substituição aos antioxidantes sintéticos, agregando valor aos subprodutos. 


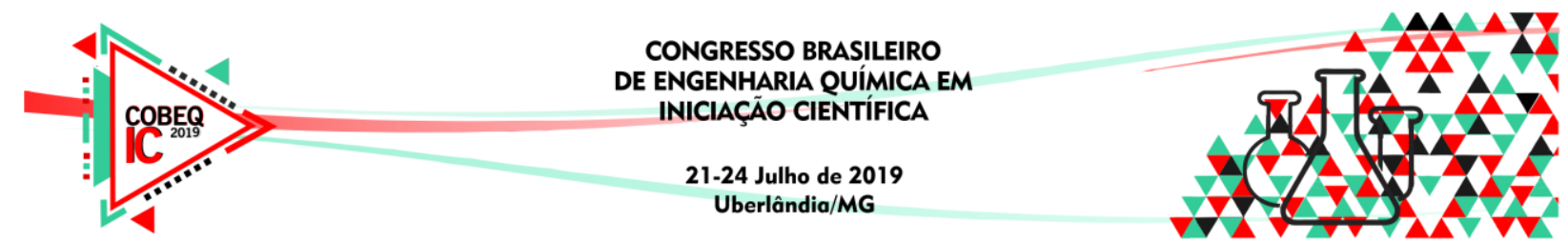

A secagem tem por definição como a operação unitária que exerce a eliminação da água, ou de outro fluído presente num material sólido. Tendo como objetivo a redução do conteúdo do fluído residual até um valor aceitavelmente baixo (Azevedo, 2017). A secagem de resíduos de frutas propõe contribuir com o desperdício, minimização dos custos de armazenamento e transporte devido à redução de peso e volume, além do projeto de obtenção de novos produtos associando-se a inovações com amplo valor nutricional e melhorando a produtividade industrial e a lucratividade.

De acordo com SANJINEZ-ARGANDOÑA et al., (2011) a modelagem matemática dos processos de secagem são importantes para otimização dos mesmos. Diante deste fato merece destaque o modelo matemático de Page (1949) muito utilizado em estudos de processos de secagem.

Neste contexto, este trabalho teve como objetivo avaliar a cinética de secagem dos resíduos agroindustriais de mamão, laranja e tomate, valorizando a possibilidade de seu reaproveitamento como um futuro subproduto (ração animal) evitando o desperdício gerado no setor agroindustrial garantindo que o produto obtido apresente maior "vida de prateleira" devido à redução do conteúdo de água.

\section{MATERIAIS E MÉTODOS}

\subsection{Preparo das amostras}

O trabalho foi desenvolvido no Laboratório de Engenharia Química da Universidade de Vassouras - RJ. Os resíduos de laranja e do mamão foram gentilmente cedidos pela empresa Sícola de processamento de frutas cristalizadas localizada na cidade de Três Rios-RJ. Os tomates foram cedidos pelo Hortifrúti Mundo Verde (Vassouras-RJ). A Figura 1 ilustra os resíduos estudados.

Figura 1 - Resíduos in natura

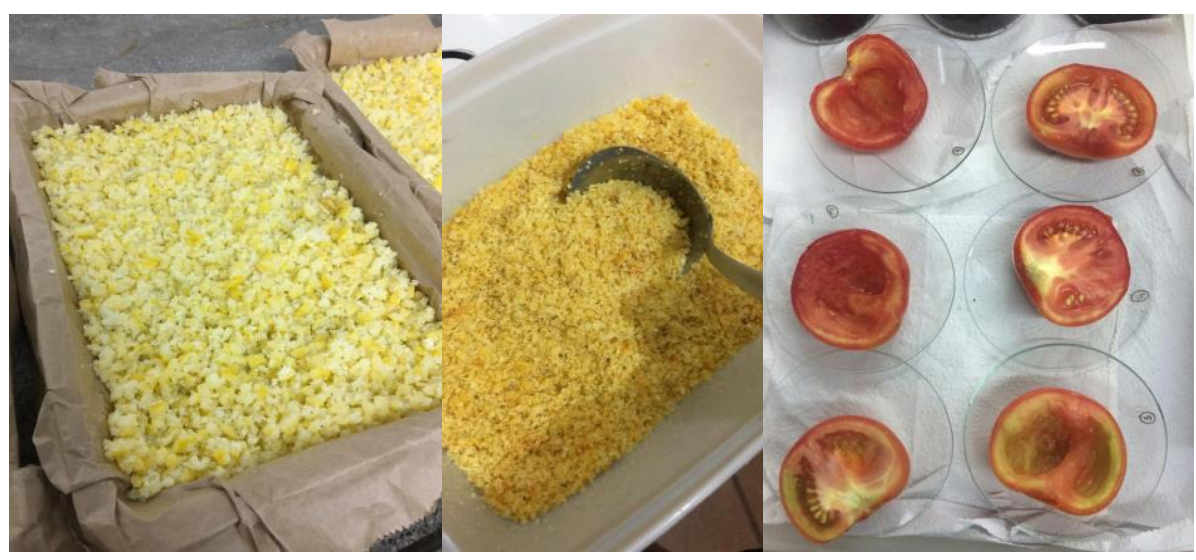

\subsection{Processo de secagem}

Os resíduos foram submetidos à secagem em estufa com circulação de ar a $60^{\circ} \mathrm{C}$. A construção das curvas de secagem foram determinadas pela umidade em base seca $(\mathrm{kg}$ de 


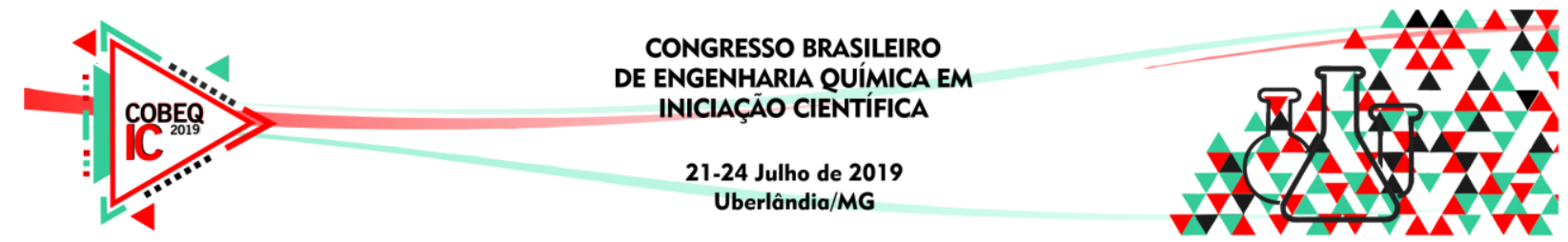

água.kg-1 sólido seco) em relação ao tempo. A taxa de secagem (dX/dt) foi calculada pela derivada da umidade em relação ao tempo descrita pela Equação 1:

$$
\frac{d x}{d t}=\frac{X_{n-1}-X_{n}}{t_{n-1}-t_{n}}
$$

Onde: $\mathrm{dX} / \mathrm{dt}=$ taxa de secagem $\left(\mathrm{kgH}_{2} \mathrm{O} / \mathrm{kgms} . \mathrm{min}\right) ; \mathrm{X}_{\mathrm{n}}$ e $\mathrm{X}_{\mathrm{n}-1}$ : umidade $\left(\mathrm{kgH}_{2} \mathrm{O} / \mathrm{kgms}\right) ; \mathrm{t}_{\mathrm{n}}$ e $\mathrm{t}_{\mathrm{n}-1}$ : tempo (min); n: número de leituras.

As cinéticas de secagem foram ajustadas pela equação de Page de acordo com a Equação 2: As constantes do modelo ( $\mathrm{k} \mathrm{e} \mathrm{n}$ ) foram estimadas através da ferramenta Solver do Excel.

$$
\frac{X_{t}-X_{\infty}}{X_{0}-X_{\infty}}=\exp \left(-k t^{n}\right)
$$

Onde: Xo, Xt e $\mathrm{X} \infty=$ umidades inicial, no tempo t e no equilíbrio, respectivamente ( $\mathrm{kgH} 2 \mathrm{O} / \mathrm{kgms}) ; \mathrm{k}$ e $\mathrm{n}=$ constantes do modelo; $\mathrm{t}=$ tempo de secagem (segundos).

Para validação do modelo matemático aplicado utilizou-se o coeficiente de determinação $\left(\mathrm{R}^{2}\right)$ e o erro médio relativo (E) de acordo com a Equação 3. O modelo é considerado adequado e representativo do processo se apresentar valores de E menores que $10 \%$.

$$
E(\%)=\frac{100}{N} \sum \frac{\left|M_{\exp }-M_{\text {pred }}\right|}{M_{\exp }}
$$

Onde: $\mathrm{M}_{\exp }=$ valores obtidos experimentalmente e $\mathrm{M}_{\text {pred }}=$ preditos pelo modelo; $\mathrm{N}=$ número de pontos experimentais.

\section{RESULTADOS E DISCUSSÃO}

A Figura 2 apresenta a perda do conteúdo de umidade das amostras ao longo do tempo e a Figura 3 apresenta o comportamento da secagem em função das taxas de secagem das amostras. 


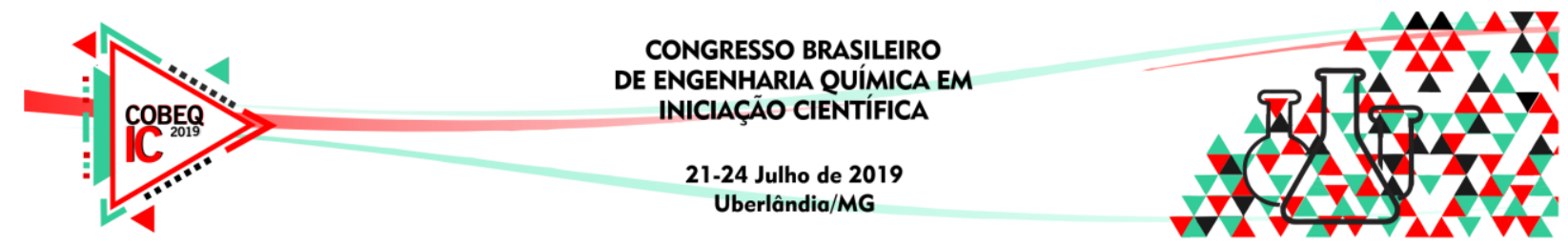

Figura 2 - Curva de secagem dos resíduos agroindustriais a $60^{\circ} \mathrm{C}$

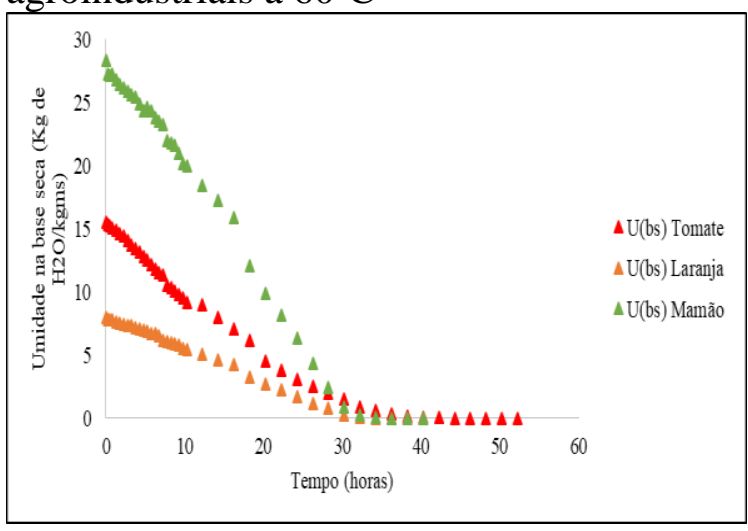

Figura 3 - Taxa de secagem dos resíduos agroindustriais $60^{\circ} \mathrm{C}$

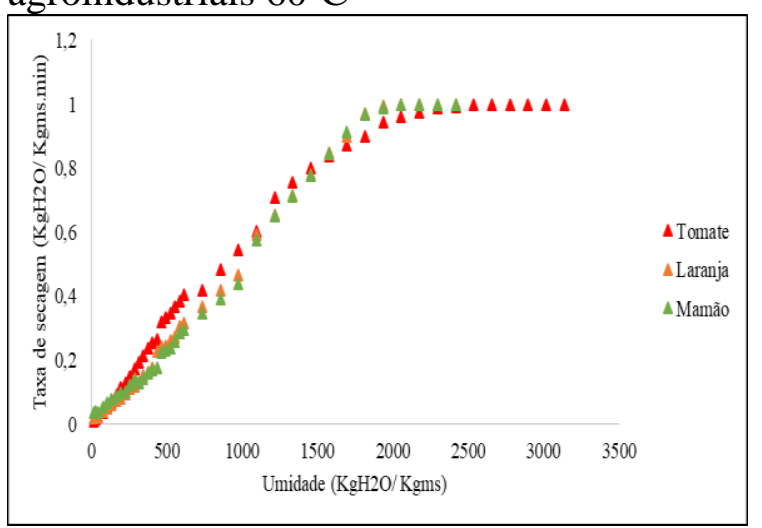

Os tempos necessários para redução do teor de água das amostras foram 36 horas para os resíduos de mamão e laranja e 50 horas para o resíduo do tomate.

De acordo com a Figura 2 observa-se que a secagem dos resíduos de mamão e laranja ocorreu em menor tempo quando comparado ao processo de secagem do tomate. Este resultado pode ser atribuído à forma de secagem, ao tempo de espera da pesagem e à umidade relativa do ar, que pode conceder, ou não, umidade às amostras enquanto armazenadas. Além da diferença de suas massas e do nível de umidade presente em cada amostra.

Em ambos os processos de secagem das três amostras dos resíduos, a taxa de secagem (Figura 3) aumenta ao longo do tempo e observa-se que até a obtenção da umidade de equilíbrio as curvas apresentam uma taxa decrescente. Segundo Sanjinez-Argandoña et al, (2011) para produtos biológicos tal comportamento pode ser explicado pela migração interna de umidade (Sanjinez-Argandoña et al, 2011).

As Figuras 4, 5 e 6 apresentam as curvas experimentais ajustadas pelo modelo de Page obtidas no processo de secagem dos resíduos de tomate, laranja e mamão, respectivamente.

Figura 4 - Modelagem matemática aplicada às curvas experimentais de secagem do resíduo do tomate

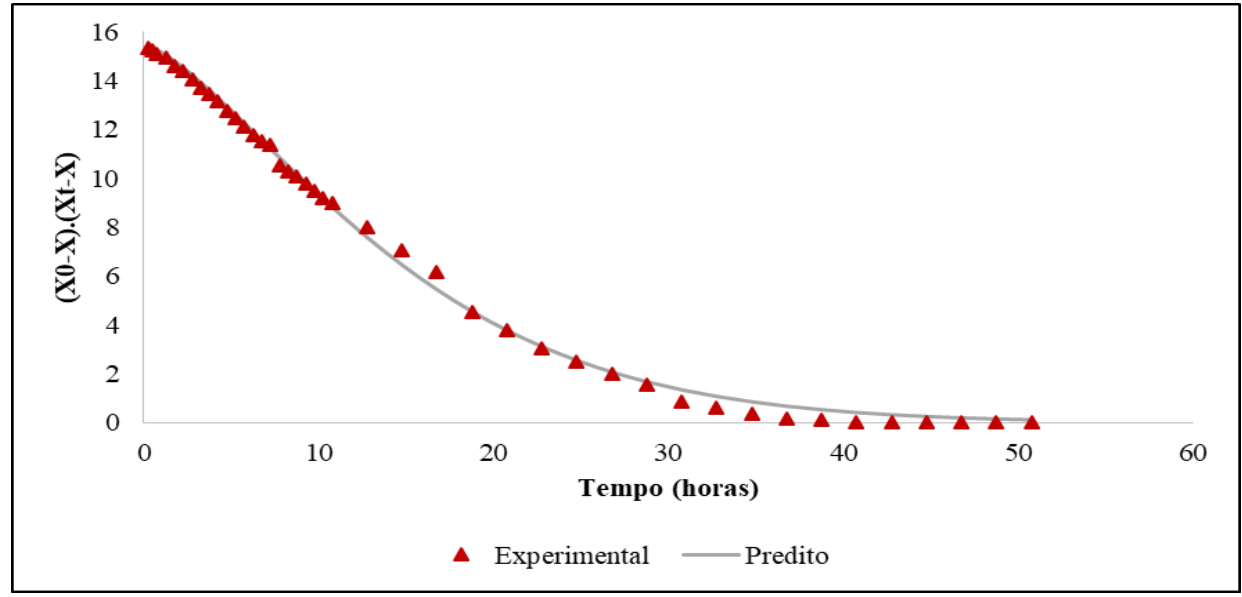




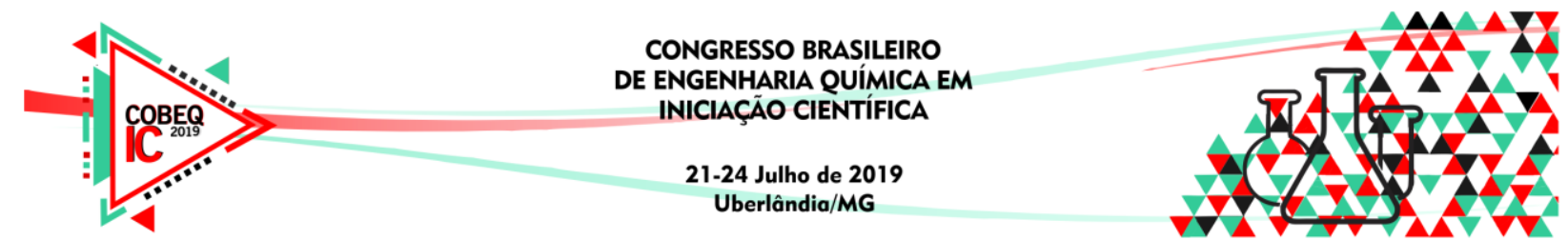

Figura 5 - Modelagem matemática aplicada às curvas experimentais de secagem do resíduo da laranja

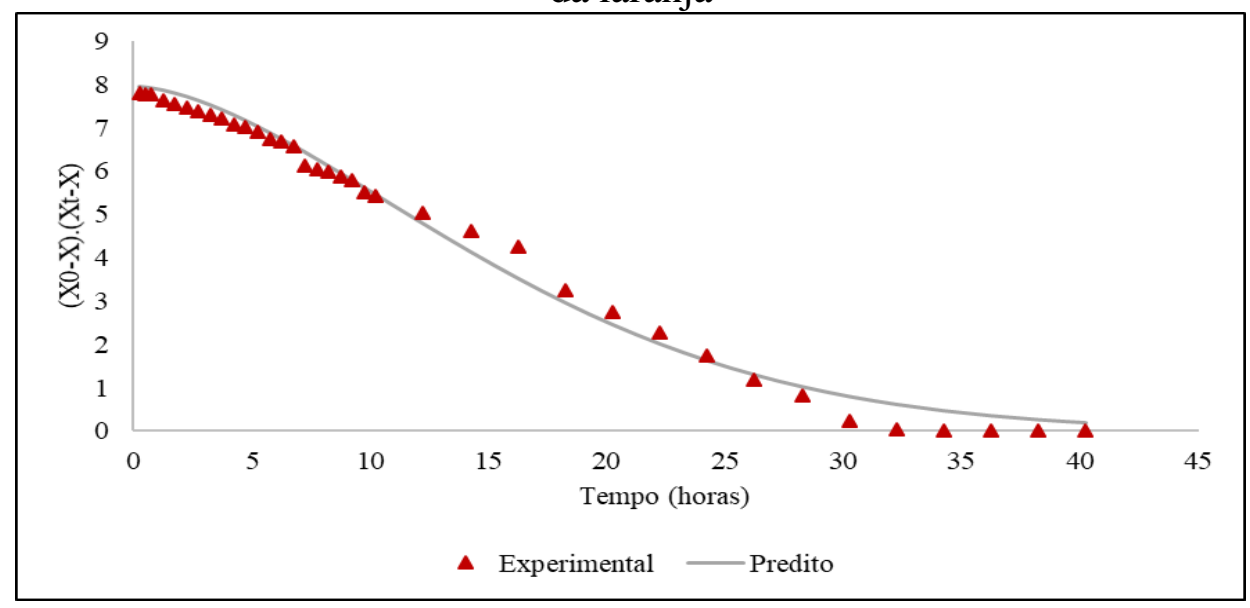

Figura 6 - Modelagem matemática aplicada às curvas experimentais de secagem do resíduo do mamão

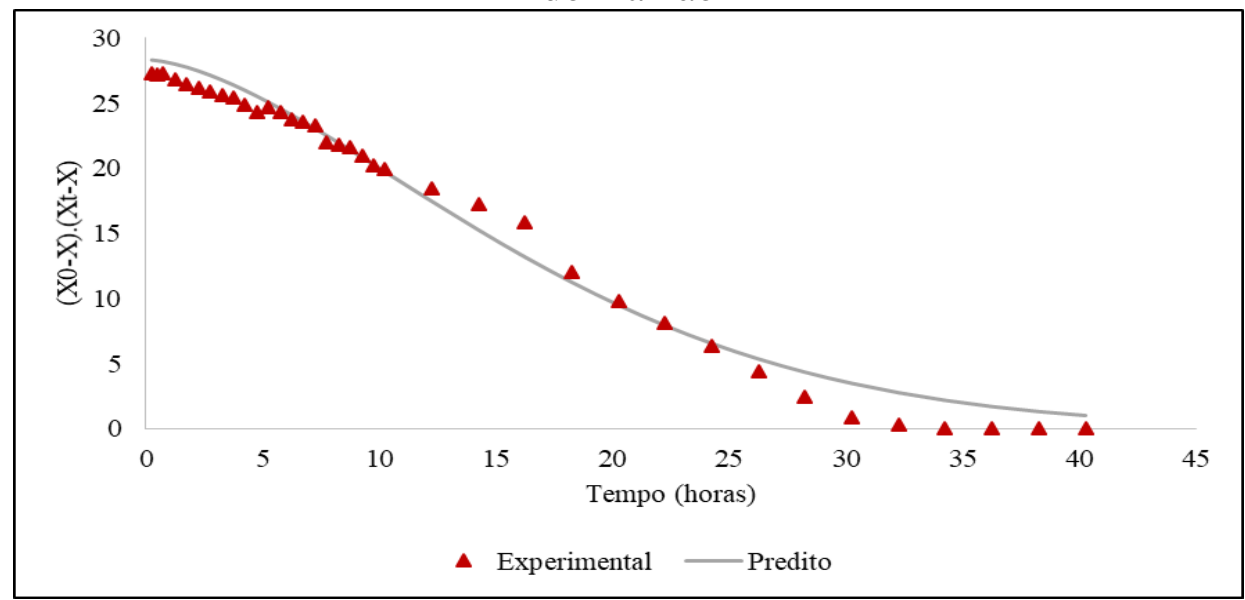

A modelagem matemática representada pela equação de Page aplicada à curva de secagem do resíduo de tomate se ajustou melhor aos dados experimentais apresentando o coeficiente de determinação $\left(\mathrm{R}^{2}\right)$ mais próximo de 1 e menor erro.

Os parâmetros estimados para os ajustes da modelagem matemática são apresentados na Tabela 1, considerando-se os diferentes tipos de resíduos sob mesma temperatura secagem, com seus respectivos coeficientes de determinação $\left(\mathrm{R}^{2}\right)$ e desvios relativos.

Tabela 1 - Parâmetros estimados e validação da modelagem matemática a partir do Modelo de Page no processo de secagem dos resíduos

\begin{tabular}{|c|c|c|c|c|}
\hline Resíduo & $\mathbf{K}\left(\right.$ minuto $\left.^{-1}\right)$ & $\mathbf{n}$ & $\mathbf{R}^{\mathbf{2}}$ & $\mathbf{E}(\boldsymbol{\%})$ \\
\hline \hline Tomate & $6,485910 \mathrm{E}-05$ & 1,401251 & 0,999123 & 0,035338 \\
\hline Laranja & $8,475920 \mathrm{E}-06$ & 1,666749 & 0,995616 & 0,061166 \\
\hline Mamão & $1,087080 \mathrm{E}-05$ & 1,621972 & 0,993935 & 0,077442 \\
\hline
\end{tabular}




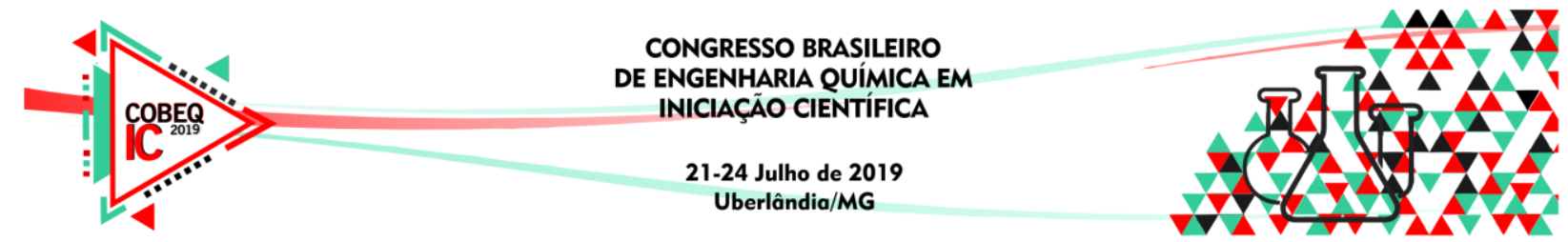

Os dados experimentais e os preditos pelo modelo foram aplicados de forma satisfatória. Ao observar os valores para o $\mathrm{R}^{2}$, conclui-se que o modelo de Page foi bem empregado neste tipo de secagem. De acordo com Bendlin (2003) alguns fatores como evaporação da água no alimento como, migração de solutos, capilaridade, deformação do produto, ligação físico-química da água às substâncias sólidas presentes no alimento, dentre outros, são fenômenos que podem apresentar ampla resistência à transferência de massa no processo de secagem.

\section{CONCLUSÃO}

De acordo com os resultados obtidos concluiu-se que o modelo de Page se ajustou com boa precisão aos dados experimentais de secagem dos resíduos estudados apresentando valores de $\mathrm{R}^{2}$ próximos a $1 \mathrm{em}$ todos os ensaios. Os resíduos de laranja e mamão apresentaram um período rápido de secagem à taxa constante em comparação a secagem do tomate. Desta forma, considera-se a secagem como um processo importante para reaproveitamento dos resíduos e sua aplicação na fabricação de um novo produto ou subproduto minimizando o desperdício dos resíduos agroindustriais.

\section{REFERÊNCIAS}

AZEVEDO, EG; ALVES, AM Collection School of Science and Technology: Separation Process Engineering. 3rd ed. Lisbon: IST Press, 2017. 795 p.

BENDLIN, R. C. S. Secagem convectiva de erva-mate (Ilex paraguariensis). 2003. $77 \mathrm{f}$. Dissertação (Mestrado em Engenharia de Alimentos)-Departamento de Engenharia Química e de Alimentos, Universidade Federal de Santa Catarina, Florianópolis, 2003.

INFANTE, J.; SELANI, M. M.; TOLEDO, N. M.V.; SILVEIRA-DINIZ, M. F.; ALENCAR, S. M.; SPOTO, M. H. F. Atividade antioxidante de resíduos agroindustriais de frutas tropicais. Brazilian Journal Food Nutricion, v.24, n.1, p.7-91, 2013.

SANJINEZ-ARGANDOÑA, Eliana Janet et al. Influência da geometria e da temperatura na cinética de secagem de tomate (Lycopersicum esculentum). Food Science and Technology (Campinas), p. 308-312, 2011.

SOUSA, M. S. B.; VIEIRA, L. M.; SILVA, M. J. M.; LIMA, A. Caracterização nutricional e compostos antioxidantes em resíduos de polpas de frutas tropicais. Ciência e Agrotecnologia, v.35, n.3, p.554-559, 2011. 